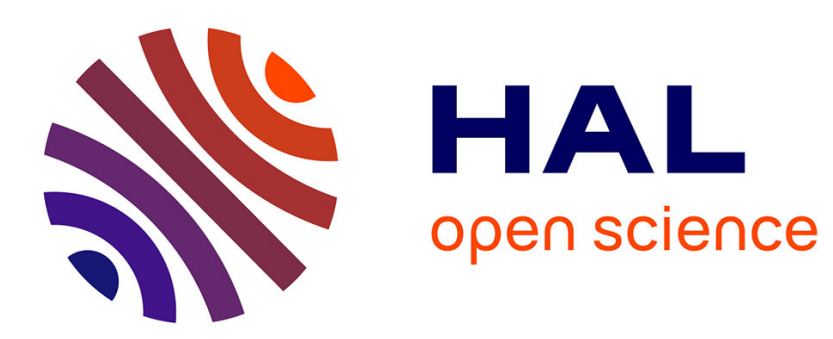

\title{
Instantons for the destabilization of the inner Solar System
}

\author{
Eric Woillez, Freddy Bouchet
}

\section{To cite this version:}

Eric Woillez, Freddy Bouchet. Instantons for the destabilization of the inner Solar System. Physical Review Letters, In press. hal-02308209v3

\section{HAL Id: hal-02308209 \\ https://hal.science/hal-02308209v3}

Submitted on 22 Jun 2020

HAL is a multi-disciplinary open access archive for the deposit and dissemination of scientific research documents, whether they are published or not. The documents may come from teaching and research institutions in France or abroad, or from public or private research centers.
L'archive ouverte pluridisciplinaire HAL, est destinée au dépôt et à la diffusion de documents scientifiques de niveau recherche, publiés ou non, émanant des établissements d'enseignement et de recherche français ou étrangers, des laboratoires publics ou privés. 


\title{
Instantons for the destabilization of the inner Solar System
}

\author{
Eric Woillez ${ }^{1,2}$ and Freddy Bouchet ${ }^{1}$ \\ ${ }^{1}$ Univ Lyon, Ens de Lyon, Univ Claude Bernard, CNRS, \\ Laboratoire de Physique, F-69342 Lyon, France. and \\ ${ }^{2}$ Department of Physics, Technion, Haifa 32000, Israel
}

\begin{abstract}
For rare events, path probabilities often concentrate close to a predictable path, called instanton. First developed in statistical physics and field theory, instantons are action minimizers in a path integral representation. For chaotic deterministic systems, where no such action is known, shall we expect path probabilities to concentrate close to an instanton? We address this question for the dynamics of the terrestrial bodies of the Solar System. It is known that the destabilization of the inner Solar System might occur with a low probability, within a few hundred million years, or billion years, through a resonance between the motions of Mercury and Jupiter perihelia. In a simple deterministic model of Mercury dynamics, we show that the first exit time of such a resonance can be computed. We predict the related instanton and demonstrate that path probabilities actually concentrate close to this instanton, for events which occur within a few hundred million years. We discuss the possible implications for the actual Solar System.
\end{abstract}

Rare events can be very important if their large impact compensate for their low probability. From a dynamical perspective, when conditioned on the occurence of a rare event, path probabilities often concentrate close to a predictable path, called instanton. This is a key and fascinating property for the dynamics of rare events and of their impact [1], which was first observed in statistical physics, for the nucleation of a classical supersaturated vapor [2]. Soon after, a similar concentration of path probabilities has been studied in gauge field theories $[3,4]$, for instance for the Yang-Mill theory. Instantons continue to have number of applications in modern statistical physics, for instance to describe excitation chains at the glass transition [5], reaction paths in chemistry [6], escape of brownian particles in soft matter [7], MHD [8] and turbulence [9-13], among many other examples. Moreover, a large effort has been pursued to develop dedicated numerical approches to compute instantons [14]. Inspired by the earlier works, action minimization have found a rigorous mathematical treatment, through the Freidlin-Wentzell large deviation theory [15] of ordinary differential equations with small noises [16].

In all those classical or quantum applications, instantons appear as action minimizers, for a saddle point evaluation of a path integral. The basic property of the instanton phenomenology is that, conditioned on the occurence of a rare event, path probabilities concentrate close to a predictable path. Fig. (1) gives an illustration of this property for a particle in a bistable potential. Shall we expect this phenomenology to be valid for systems for which the Freidlin-Wentzell action (Please note that the word "action" refers to the path integral of large deviation theory, and has nothing to do with the classical action of analytical mechanics that can be written for Hamiltonian dynamics). does not exist in the first place, for instance chaotic deterministic systems? The main aim of this work is to open this fascinating question for a paradigmatic problem in the history of physics: the dynamics of the Solar System. Shall we expect an instanton phenomenology for rare events that shaped or

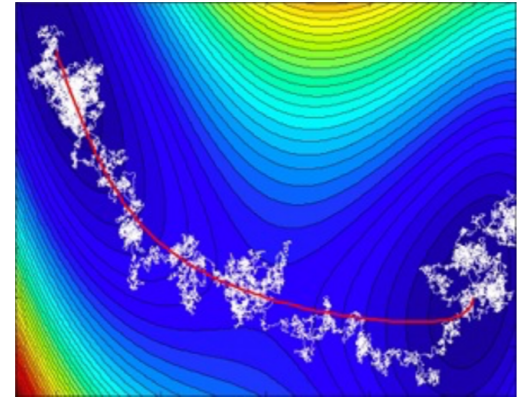

FIG. 1: Instanton for a Brownian particle in a bistable potential. The particle's trajectory from one attractor to another (white line) closely follows the minimum action path (instanton, red line), up to thermal fluctuations. The level curves of the potential are displayed in the background, with the color scale giving the potential's height (courtesy Eric VandenEijnden).

will shape the Solar System history?

The discovery that our solar system is chaotic with a Lyapunov time of about 5 million years [17-19] has disproved the previous belief that planetary motion would be predictable with any desired degree of precision. On the contrary, chaotic motion sets an horizon of predictability of a few tens of million years for the solar system. Even more striking has been the discovery that about $1 \%$ of the trajectories in the Solar System lead to collisions between planets, or between planets and the Sun within 5 billion years [20]. As shown numerically, chaotic disintegration of the inner solar system (i.e. the four terrestrial planets) always happens through a resonance between the motion of Mercury's and Jupiter's perihelia [20-23], related to a large increase in Mercury's eccentricity. Stochastic perturbation to planetary motion exists, for instance through the chaotic motion of the asteroid belt, but is too weak to be responsible for the rare destabilizations of the inner solar system [22, 24]. Instead, stochasticity in the solar system appears because of the development of internal deterministic chaos [22]. 
Does an instanton phenomenology exist for the rare destabilization of the Solar System? Our first result will be obtained within a simplified model of Mercury's dynamics [25]. We predict for this model the probability distribution of the first destabilization time, the instanton paths, and check the instanton phenomenology.

The secular dynamics describes the planetary motion averaged over fast orbital motion. The secular dynamics Hamiltonian is

$$
H(\mathbf{I}, \boldsymbol{\Phi})=H_{\text {int }}(\mathbf{I})+\sum_{\mathbf{k} \in \mathbb{Z}^{16}} A^{k}(\mathbf{I}) \cos (\mathbf{k .} \mathbf{\Phi}),
$$

where $(\mathbf{I}, \boldsymbol{\Phi})$ is the canonical set of Poincaré action-angle variables for the 8 planets, $\mathbf{k}$ is a vector of integers, and the coefficients $A^{k}$ are functions of the action variables only (see e.g [26] for the explicit expression of $H$ to forth order in planetary eccentricities and inclinations). We will study Mercury's possible destabilization in the framework of a simplified model proposed by Batygin and col. [25]. This model should be seen as a minimal model retaining the relevant interactions leading to destabilization of the inner Solar System but is not expected to describe quantitatively the inner Solar System.

The approximations of [25] consist in keeping only the degrees of freedom of a massless Mercury in the Hamiltonian (1), and replace all other action-angle variables by their quasiperiodic approximation. Assuming moreover that only a small number of periodic terms in Eq. (1) significantly affect the long-term secular motion of Mercury [21, 23, 25, 27], Mercury's simplified Hamiltonian is

$$
\begin{aligned}
H= & H_{\text {int }}(I, J)+E_{2} \sqrt{I} \cos (\varphi)+S_{2} \sqrt{J} \cos (\psi) \\
& +E_{T} \sqrt{I} \cos \left(\varphi+\left(g_{2}-g_{5}\right) t+\beta\right),
\end{aligned}
$$

where $\varphi$ and $\psi$ are the canonical angles conjugated to $I=1-\sqrt{1-e^{2}}$ and $J=\sqrt{1-e^{2}}(1-\cos i)$ respectively, and $e$ and $i$ are Mercury's eccentricity and inclination [25]. $g_{5}, g_{2}$ and $s_{2}$ are frequencies involved in the quasiperiodic decomposition of the motion of Jupiter $\left(g_{5}\right)$ and Venus $\left(g_{2}\right.$ and $\left.s_{2}\right)$. The numerical values for the other coefficients in Eq. (2) are given in appendix.

A slow variable for Mercury's dynamics: We first show how a slow variable can be built from the dynamics defined by the Hamiltonian (2). In Eq. (2), $H_{\text {int }}$ only depends on the actions. Would the total Hamiltonian be reduced to this part, the actions would be constant and the canonical angles would simply grow linearly with time according to Hamilton's equations

$$
\left\{\begin{array}{l}
\dot{\varphi}(t)=\frac{\partial H_{i n t}}{\partial I}=-g_{1}(I, J)+g_{5} \\
\dot{\psi}(t)=\frac{\partial H_{i n t}}{\partial J}=-s_{1}(I, J)+s_{2}
\end{array}\right.
$$

The fundamental frequencies $g_{1}(I, J)$ and $s_{1}(I, J)$ describe Mercury's perihelion precession at frequency $g_{1}$, and its orbital plane oscillations with respect to the invariant reference plane, at frequency $s_{1}$. For the model (2), $g_{1}$ value is about $5.7^{\prime \prime} / y r$, corresponding to a period of about 227000 years (This value is actually specific of our model. The current value of $g_{1}$ for the real Solar System would be about 5.60"/yr).

Through the chaotic dynamics of (2), the fundamental frequencies $\left\{g_{1}, s_{1}\right\}$ change over time. Mercury's secular motion might enter into resonance with the external periodic forcing if $g_{1}$ or $s_{1}$ comes close to one of the frequencies $g_{5}, g_{2}$ or $s_{2}$. In particular, the Mercury-Jupiter perihelion resonance, between $g_{1}$ and $g_{5}$, might trigger Mercury's destabilization [20-23]. The three curves of equations $g_{1}(I, J)=g_{5}, s_{1}(I, J)=s_{2}$ and $g_{1}(I, J)=g_{2}$ can be represented in the $(I, J)$ plane, together with the current values of Mercury's action variables. We obtain in Fig. (2) the so-called "resonance map" which is now widely used for weakly non-integrable systems [29, 30]. We write (2) as $H=\widetilde{H}+H_{\text {pert }}$, with

$$
\begin{gathered}
\widetilde{H}=H_{i n t}+E_{2} \sqrt{I} \cos (\varphi)+S_{2} \sqrt{J} \cos (\psi), \\
H_{\text {pert }}=E_{T} \sqrt{I} \cos \left(\varphi+\left(g_{2}-g_{5}\right) t+\beta\right) .
\end{gathered}
$$

The term $H_{\text {pert }}$ given by (5) creates a weak perturbation for Mercury's long-term evolution. To find the order of magnitude at which $H_{\text {pert }}$ affects the long-term dynamics of Mercury, we employ Lie transform methods [30] with the software TRIP (TRIP is a general computer algebra system dedicated to celestial mechanics developed at the IMCCE (Copyright 1988-2019, J. Laskar ASD/IMCCE/CNRS). TRIP is particularly efficient to handle series with a large number of terms like those usually appearing in Lie transforms. ).

There exists new action-angle variables and a canonical transformation such that Mercury's Hamiltonian can be put in the form

$$
H^{\prime}=\widetilde{H}^{\prime}\left(I^{\prime}, J^{\prime}, \varphi^{\prime}, \psi^{\prime}\right)+H_{p e r t}^{\prime}\left(I^{\prime}, J^{\prime}, \varphi^{\prime}, \psi^{\prime},\left(g_{2}-g_{5}\right) t\right),
$$

where the order of magnitude of $H_{\text {pert }}^{\prime}$ is much smaller than $H_{\text {pert }}$. The Lie transform creates periodic terms in $H_{\text {pert }}^{\prime}$ that contain new combinations of the angles $\varphi^{\prime}, \psi^{\prime}$ and $\left(g_{2}-g_{5}\right) t$ (given in the appendix). The difference between $H_{\text {pert }}$ and $H_{\text {pert }}^{\prime}$ is that the angular terms of the latter are resonant, which means that their frequencies can vanish. The existence of such resonant terms, even of small amplitude, generate long-term chaotic motion.

The Hamiltonian (6) defines a dynamical system with two well separated time scales. On a time scale of the order of $\frac{1}{g_{1}}$, the action-angle variables evolve according to Hamilton's equations of motion. The flow is chaotic with a Lyapunov time $\tau_{L}$ of the order of one million years [25]. $\widetilde{H}^{\prime}$ evolution

$$
\dot{\tilde{H}}^{\prime}=\left\{H_{\text {pert }}^{\prime}, \widetilde{H}^{\prime}\right\}
$$

sets a new time scale. In Eq. (7), the notation \{\} represents the canonical Poisson brackets. Eq. (7). shows that $\widetilde{H}^{\prime}$ is a slow variable, because its time evolution is driven by $H_{\text {pert }}^{\prime} \ll H_{\text {pert }}$. As will become clear in the 


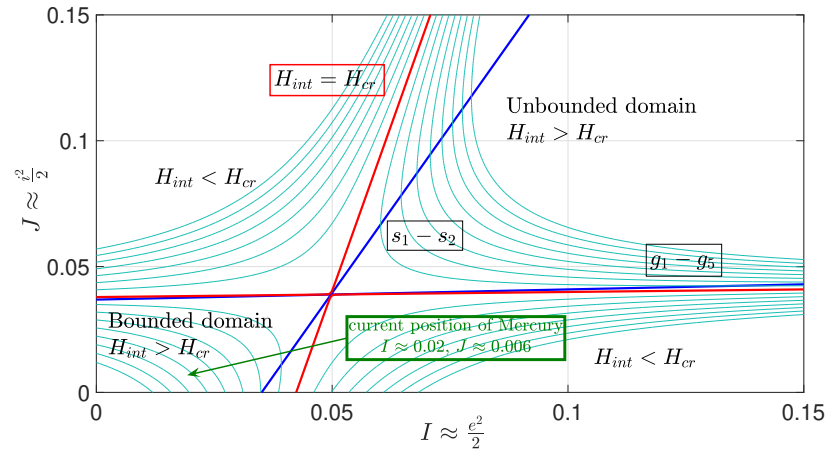

FIG. 2: Level curves of $H_{\text {int }}(I, J)$ in action space. The surface defined by $H_{\text {int }}(I, J)$ has the structure of a saddle. Mercury currently satisfies $H_{\text {int }}>H_{c r}$ and is located in the bounded domain. For destabilization to occur, Mercury has to cross the saddle and enter the unbounded domain.

following, $\widetilde{H}^{\prime}$ remains almost constant on the fast time scale, and has only significant variations on a timescale of a few hundred million years.

Diffusion of the slow variable: The theory of white noise limit for slow-fast dynamical systems (see e.g. [31]) suggests that on a timescale much larger than $\tau_{L}$, Eq. (7) is equivalent to a diffusion process. This limit is valid assuming that the variations of $\widetilde{H}^{\prime}$ on the timescale $\tau_{L}$ are sufficiently small. Two additional phenomenological approximations can be made: first, numerical simulations performed with Eq. (7) show that the drift is very small compared to the diffusion coefficient, and can be neglected. Second, the range of $\widetilde{H}^{\prime}$ values before destabilization is small, and the diffusion coefficient can be considered as constant. The long-term evolution of $\widetilde{H}^{\prime}$ can thus be modeled by the standard Brownian motion

$$
\dot{\tilde{H}}^{\prime}=\sqrt{D} \xi(t)
$$

where $\xi(t)$ is the Gaussian white noise with correlation function $\left\langle\xi(t) \xi\left(t^{\prime}\right)\right\rangle=\delta\left(t-t^{\prime}\right)$. Unfortunately, the exact expression for $D$ involves the full correlation function of the Hamiltonian flow defined by $\widetilde{H}^{\prime}$. It is too intricate to be useful in practice. Starting from the formal expression, it is shown in the appendix that an order of magnitude is

$$
D \approx 2\left|H_{\text {pert }}\right|^{6} \tau_{L} /|\widetilde{H}|^{4},
$$

where $|\widetilde{H}|$ and $\left|H_{\text {pert }}\right|$ are orders of magnitude of (5) and (4) respectively. Eq. (9) is our first important result. Evaluating Eq. (9) gives $D \approx 7.2 \times 10^{-7} \mathrm{Myr}^{-3}$. The associated diffusion time scale for $\widetilde{H}^{\prime}$ is evaluated to one billion years. Those results justifies the self-consistency of the choice for the slow variable.

Distribution of the first destabilization times of Mercury: We now discuss qualitatively the implications of the existence of a slow variable for Mercury's destabilization. This discussion is best understood looking at the level

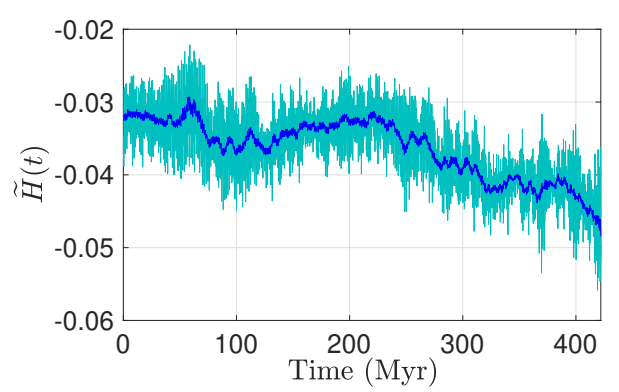

FIG. 3: A trajectory $\widetilde{H}(t)$ (cyan) compared to its local time average $h(t)$ (blue). The local time averaging of $\widetilde{H}(t)$ suppresses the fast oscillations that do not correspond to longterm variations. For the long-term chaotic dynamics, $h(t)$ is an slow variable that follows a standard Brownian motion.

curves of $H_{\text {int }}(I, J)$ in action space displayed in Fig. (2). It can be seen that the landscape defined by $H_{\text {int }}(I, J)$ has the topology of a saddle. The saddle is exactly located at the intersection between the two resonances $g_{1}-g_{5}$ and $s_{1}-s_{2}$, with the value $H_{i n t}=H_{c r}$. The domain of equation $H_{\text {int }}(I, J) \geq H_{c r}$ has two disjoint components, one bounded (bottom left) and the other unbounded (top right), only connected by the saddle point $\left(I_{c r}, J_{c r}\right)$. The initial orbital parameters of Mercury $e$ and $i$ are located in the bounded domain, which implies that the short-time orbital fluctuations are restricted to this part of phase space. When $H_{\text {int }}$ reaches the value $H_{c r}$, Mercury can cross the saddle and enter the unbounded domain of phase space. This latter event defines Mercury's destabilization.

We explain in the appendix how the above simple criterion translates into an equivalent criterion for $\widetilde{H}^{\prime}$ : there exists a threshold $h_{c r}$ for which the first destabilization time exactly corresponds to the first hitting time of $\widetilde{H}^{\prime}$ to $h_{c r}$.

The full expression of $\widetilde{H}^{\prime}$ is an intricate serie composed of a large number of periodic terms of small amplitude, which explicit expression is difficult to handle. Following [25], we prefer to use in practice the local time average $h(t)=\langle\widetilde{H}\rangle_{[t-\theta, t+\theta]}$ as an approximation of $\widetilde{H}^{\prime}$, which is much simpler to implement numerically. The time frame $\theta$ has to be much larger than the frequency of the fast variations of $\widetilde{H}$ given by the frequency $g_{2}-g_{5}$ according to Eq. (5). As an example, the time variations of $\widetilde{H}(t)$ compared to those of $h(t)$ is displayed in Fig. (3) with $\theta=2$ Myr. We then identify the diffusion Eq. (8) for $\widetilde{H}^{\prime}$ and that for $h$.

Tracking numerically the value of $h(t)$ of trajectories leading to destabilization confirms that the distribution $h(\tau)$ (where $\tau$ is the destabilization time) is peaked at the value $h_{c r}=-0.048$, which can thus be identified as the destabilization threshold. We must also add a reflective boundary for a upper value $h_{\text {sup }}$, accounting for the fact that the chaotic region of phase space before destabilization is bounded. Destabilization of Mercury occurs when 


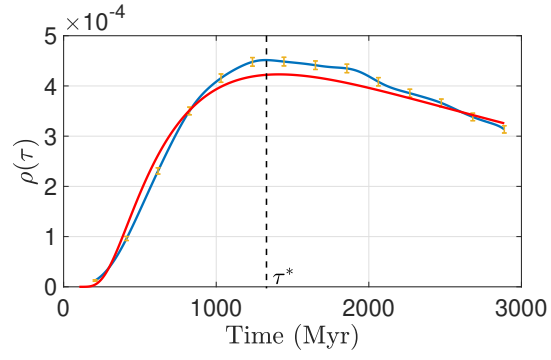

FIG. 4: Probability distribution of Mercury's first destabilization time. The distribution of Mercury's first destabilization time is computed with a direct numerical simulation (blue curve) and with the theoretical prediction of the diffusive model Eq. (8) (red curve).

the Brownian motion defined by $h(t)$ reaches $h_{c r}$. For a standard Brownian motion, the distribution $\rho(\tau)$ of first hitting times of the value $h_{c r}$ can be derived exactly (see appendix). The latter is displayed in Fig. (4), together with the distribution obtained from direct numerical simulations of Hamilton's equations. $D$ is the only fitting parameter and can be estimated as $D \approx 9.6 * 10^{-7} \mathrm{Myr}^{-3}$. Using this value, Fig. (4) shows that the diffusive model Eq. (8) gives a excellent qualitative agreement with the direct numerical simulations. The fitted value of $D$ is also in agreement with Eq. (9) and its order of magnitude $D \approx 7.2 \times 10^{-7} \mathrm{Myr}^{-3}$.

Instanton paths for Mercury: We now focus on the probability that Mercury's orbit is destabilized in short times $\tau_{L} \ll \tau \ll \tau^{*}$, where $\tau^{*}$ is the maximum of $\rho(\tau)$. The probability $\mathbb{P}(\tau)=\int_{0}^{\tau} \rho_{t h}\left(\tau^{\prime}\right) \mathrm{d} \tau^{\prime}$ that the destabilization of Mercury's orbit occurs in a time shorter than $\tau$ is dominated at short times by the exponential term $\rho(\tau) \underset{\tau \rightarrow 0}{\asymp} e^{-\frac{\bar{\tau}}{\tau}}$, where $\bar{\tau}=\frac{\left(h_{0}-h_{c r}\right)^{2}}{4 D} \approx 1.56 * 10^{9}$ years.

The exponential growth is the signature that shortterm destabilizations of Mercury are rare events. The slow variable $h(t)$, conditioned on the fact that destabilization occurs at a given time $\tau$, is predictable by the instanton path. The dynamics of $h(t)$ is simple enough such that the instanton path can be computed exactly: it is the straight path starting at $h(0)$ and reaching $h_{c r}$ at time $\tau$. We can even obtain a more precise result, namely the exact expressions for the average and the variance of all trajectories destabilized in a given time $\tau$. The theoretical and numerical results for $\tau=445$ million years is displayed in Fig. (5). The middle blue curve displays the averaged trajectory obtained through direct numerical averaging of all trajectories leading to destabilization at time $\tau$. In addition, the upper and lower blue curves display the variance of the trajectories ensemble, and show how the trajectories depart from the most probable trajectory. We have superimposed three red curves that represent the average and variance of the probability distribution $\mathbf{P}\left[h, t \mid\left(h_{c r}, \tau\right),\left(h_{0}, 0\right)\right]$ to observe the value $h$ at time $t$, with the constrain $h(\tau)=h_{c r}$, for the standard Brownian motion $h(t)$.

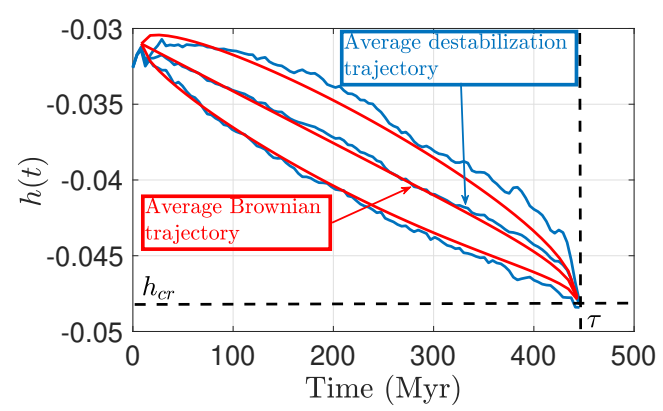

FIG. 5: Prediction of the trajectory leading to Mercury's short-term destabilization. The blue curves display the average trajectory and the variance of the trajectories leading to a destabilization at $\tau=445$ million years, obtained with direct numerical simulations. The red curves display the same quantities obtained with the theory of rare events (prediction of the instanton, see appendix).

The agreement between the diffusive model of $h$ and Mercury's dynamics can be considered as excellent, notwithstanding the small discrepancy at short times coming from the finite correlation time of Mercury's secular dynamics. This is a second confirmation that the diffusive model for the slow variable is consistent both for the prediction of Mercury's first destabilization time distribution, and for the prediction of instantons. However, we note that the simple picture of a straight-line instanton is bound to the validity of the diffusive limit used to derive Eq. (8). The simple approach described in this paper would fail if, for example, the averaged dynamics of $\widetilde{H}^{\prime}$ would not be negligible.

Within the Batygin-Morbidelli-Holman dynamics, a reduced model of the inner Solar System with deterministic chaos, we have shown that the first exit time for a Mercury-Jupiter resonance can be computed from an effective stochastic diffusion. We have gone beyond this result, and we predicted the related instanton and demonstrated that path probabilities actually concentrate close to this instanton, for events which occur within a few hundred million years. For the Batygin-Morbidelli-Holman model, both the instanton and the variance of the trajectories leading to Mercury's destabilization can be computed exactly. While the model contains some of the features of the inner Solar System dynamics, it neglects others. Clearly, this model should not be expected to quantitatively predict first exit times for the actual Solar System. Nevertheless, the instanton phenomenology is robust to more complex dynamics. Even if the secular dynamics of the real Mercury cannot be reduced to a simple diffusion model as done in this paper, our striking results suggest that the destabilization of the Solar System might indeed occur though an instanton phenomenology. Our work opens this question, which should be addressed within other models, that have to be realistic enough for describing faithfully the actual dynamical mechanisms, 
but simple enough for a proper statistical study.

\section{Acknowledgments}

We are highly indebted to F. Mogavero and J. Laskar for their constant help all along this work and in particular for having shared with us the private version of
TRIP and the quasiperiodic decomposition of planetary motion. We also thank C. Batygin and A. Morbidelli for having shared their previous results with us, and for interesting discussions. The research leading to these results has received funding from the European Research Council under the European Union's seventh Framework Program (FP7/2007-2013 Grant Agreement No. 616811).
[1] Francesco Ragone, Jeroen Wouters, and Freddy Bouchet. Computation of extreme heat waves in climate models using a large deviation algorithm. Proceedings of the $\mathrm{Na}$ tional Academy of Sciences 115, 24 (2018).

[2] J. S. Langer. Theory of the condensation point. Annals of Physics 41, 108 (1967).

[3] Sidney R. Coleman. The Uses of Instantons. Subnucl. Ser. 15, 805 (1979). [,382(1978)].

[4] Jean Zinn-Justin. Quantum field theory and critical phenomena. Clarendon Press 1996.

[5] JS Langer. Excitation chains at the glass transition. Physical review letters $\mathbf{9 7}, 115704$ (2006).

[6] N. G. van Kampen. Stochastic processes in physics and chemistry. North-Holland personal library. Elsevier Amsterdam ; Boston 3rd ed edition 2007.

[7] E. Woillez, Y. Zhao, Y. Kafri, V. Lecomte, and J. Tailleur. Activated Escape of a Self-Propelled Particle from a Metastable State. Phys. Rev. Lett. 122, 258001 (2019).

[8] M. Berhanu, R. Monchaux, S. Fauve, N. Mordant, F. Petrelis, A. Chiffaudel, F. Daviaud, B. Dubrulle, L. Marie, F. Ravelet, M. Bourgoin, P. Odier, J. . Pinton, and R. Volk. Magnetic field reversals in an experimental turbulent dynamo. Eur. Phys. Lett. (2007).

[9] Tobias Grafke, Rainer Grauer, and Tobias Schäfer. Instanton filtering for the stochastic Burgers equation. Journal of Physics A: Mathematical and Theoretical 46, 062002 (2013).

[10] Jason Laurie and Freddy Bouchet. Computation of rare transitions in the barotropic quasi-geostrophic equations. NEW JOURNAL OF PHYSICS 17 (2015).

[11] Tobias Grafke, Rainer Grauer, and Stephan Schindel. Efficient computation of instantons for multi-dimensional turbulent flows with large scale forcing. Communications in Computational Physics 18, 577 (2015).

[12] Freddy Bouchet, Joran Rolland, and Eric Simonnet. Rare event algorithm links transitions in turbulent flows with activated nucleations. Physical review letters 122, 074502 (2019).

[13] Giovanni Dematteis, Tobias Grafke, and Eric VandenEijnden. Rogue waves and large deviations in deep sea. Proceedings of the National Academy of Sciences 115, 855 (2018).

[14] Tobias Grafke and Eric Vanden-Eijnden. Numerical computation of rare events via large deviation theory. Chaos: An Interdisciplinary Journal of Nonlinear Science $\mathbf{2 9}$ 063118 (2019).

[15] M.I. Freidlin and A.D. Wentzell. Random Perturbations of Dynamical Systems. Springer-Verlag, 3dr ed. New York 2012.

[16] R Graham. Macroscopic potentials, bifurcations and noise in dissipative systems. In Fluctuations and Stochas- tic Phenomena in Condensed Matter pages 1. Springer 1987.

[17] Jacques Laskar. A numerical experiment on the chaotic behaviour of the solar system. Nature 338, 237 (1989).

[18] Jacques Laskar. The chaotic motion of the solar system: a numerical estimate of the size of the chaotic zones. Icarus 88, 266 (1990).

[19] GJ Sussman and J. Wisdom. Chaotic evolution of the solar system. Science 257, 56 (1992).

[20] Jacques Laskar and Mickaël Gastineau. Existence of collisional trajectories of Mercury, Mars and Venus with the Earth. Nature 459, 817 (2009).

[21] Konstantin Batygin and Gregory Laughlin. On the dynamical stability of the solar system. The Astrophysical Journal 683, 1207 (2008).

[22] Jacques Laskar. Chaotic diffusion in the Solar System. Icarus 196, 1 (2008).

[23] Gwenaël Boué, Jacques Laskar, and François Farago. A simple model of the chaotic eccentricity of Mercury. Astronomy $\& 5$ Astrophysics 548, A43 (2012).

[24] Eric Woillez and Freddy Bouchet. Long-term influence of asteroids on planet longitudes and chaotic dynamics of the solar system. Astronomy \& Astrophysics 607, A62 (2017).

[25] Konstantin Batygin, Alessandro Morbidelli, and Matthew J Holman. Chaotic disintegration of the inner Solar System. The Astrophysical Journal 799, 120 (2015).

[26] Jacques Laskar and Philippe Robutel. Stability of the planetary three-body problem. Celestial Mechanics and Dynamical Astronomy 62, 193 (1995).

[27] Yoram Lithwick and Yanqin Wu. Theory of secular chaos and Mercury's orbit. The Astrophysical Journal 739, 31 (2011).

[28] See Supplementary Material [url] for the values of coefficients in Eqs. (2) and (3), the list of third order resonances in Eq. (6), details about the derivation of Eq. (9), the critical threshold for the slow variable, and the explicit expression for the distribution of first exit time of a Brownian motion from a bounded domain.

[29] Jacques Laskar. Frequency analysis for multi-dimensional systems. Global dynamics and diffusion. Physica D: Nonlinear Phenomena 67, 257 (1993).

[30] Alessandro Morbidelli. Modern celestial mechanics: aspects of solar system dynamics. 2002.

[31] Crispin W Gardiner. Stochastic methods. SpringerVerlag, Berlin-Heidelberg-New York-Tokyo 1985.

[32] Alessandro Morbidelli and Antonio Giorgilli. On the role of high order resonances in normal forms and in separatrix splitting. Physica D: Nonlinear Phenomena 102, 195 (1997). 
[33] Gongjie Li and Konstantin Batygin. On the spin-axis 790, 69 (2014).

\section{COEFFICIENTS OF MERCURY'S HAMILTONIAN}

Mercury's simplified Hamiltonian is given by Eq. (2) in the main text

$$
\begin{aligned}
H= & H_{i n t}(I, J) \\
& +E_{2} \sqrt{I} \cos (\varphi)+S_{2} \sqrt{J} \cos (\psi) \\
& +E_{T} \sqrt{I} \cos \left(\varphi+\left(g_{2}-g_{5}\right) t+\beta\right),
\end{aligned}
$$

with

$$
H_{\text {int }}(I, J)=\left(E_{1}+g_{5}\right) I+E_{3} I^{2}+\left(S_{1}+s_{2}\right) J+S_{3} J^{2}+F_{E S} I J
$$

We give in table (I) the numerical value for the coefficients.

\begin{tabular}{|c|c|}
\hline$E_{1}+g_{5}$ & -1.68964 \\
\hline$E_{3}$ & -0.905766 \\
\hline$S_{1}+s_{2}$ & -1.54396 \\
\hline$S_{3}$ & -8.55372 \\
\hline$F_{E S}$ & 45.2859 \\
\hline$E_{2}$ & 0.0730504 \\
\hline$S_{2}$ & 0.0421457 \\
\hline$E_{T}$ & 0.0643625 \\
\hline$g_{2}$ & 7.4559 \\
\hline$g_{5}$ & 4.2575 \\
\hline$s_{2}$ & -6.57 \\
\hline$\beta$ & $169.86^{\circ}$ \\
\hline
\end{tabular}

TABLE I: Numerical value (in arcsec/yr) of the coefficients of the Hamiltonian (2) in the main text. The phase $\beta$ is expressed in degrees.

\section{DIFFUSION PROCESS FOR THE SLOW VARIABLE}

The present section is quite technical. We derive the formal expression of the diffusion coefficient $D$ in Eq. (8) using Lie transform methods, and we explain how a good order of magnitude for $D$ can be deduced from the result. The computation have been done with the software TRIP developed at the IMCCE by Jacques Laskar and Mickael Gastineau (https://www.imcce.fr/trip/), which is precisely devoted to the computation of series in celestial mechanics.

\section{A. List of third order resonances}

We start from the Hamiltonian (10) (Eq. (2) of the main text), that we decompose in two parts

$$
H=\widetilde{H}(I, J, \varphi, \psi)+\epsilon H_{p e r t}(I, J, \varphi, \psi, g t),,
$$

where $g=g_{2}-g_{5}$, with $\widetilde{H}$ and $H_{\text {pert }}$ given by Eqs. (4-5) of the main text

$$
\widetilde{H}=H_{\text {int }}(I, J)+E_{2} \sqrt{I} \cos (\varphi)+S_{2} \sqrt{J} \cos (\psi)
$$

and

$$
H_{\text {pert }}=E_{T} \sqrt{I} \cos \left(\varphi+\left(g_{2}-g_{5}\right) t+\beta\right) .
$$




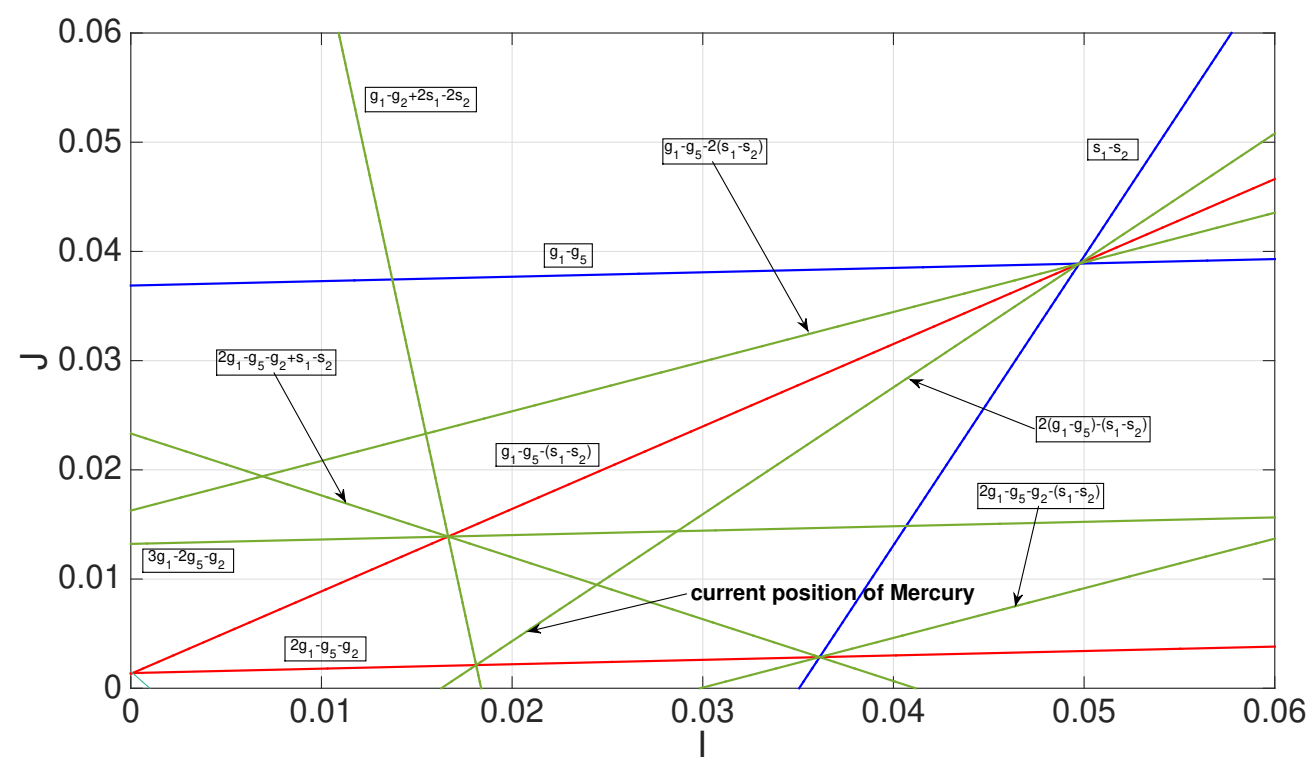

FIG. 6: The resonance map in action space. The blue lines represent the first order resonances, the red lines to the second order resonances, and the green lines to third order. Mercury's current position is close to an intersection of many third order resonances.

The parameter $\epsilon$ in Eq. (12) is used below to define a hierarchy of Lie transforms, but is set to one at the end of the calculation. Table (I) gives the values to compute the order of magnitude of $|\widetilde{H}|$ and $\left|H_{\text {pert }}\right|$ respectively. We find $|\widetilde{H}| \approx 3 \times 10^{-2} \operatorname{arcsec} / \mathrm{yr}$, and $\left|H_{\text {pert }}\right| \approx 9 \times 10^{-3} \operatorname{arcsec} / \mathrm{yr}$. We perform a canonical change of variables $\{I, J, \varphi, \psi\} \rightarrow\left\{I^{\prime}, J^{\prime}, \varphi^{\prime}, \psi^{\prime}\right\}$ with Lie transform methods to integrate the term $H_{\text {pert }}$ and all non-resonant harmonics. The procedure is described with all details in many references [30,32], but we explain briefly below the general principle.

The canonical transformation is given by a function $\chi(I, J, \varphi, \psi)$ such that the new Hamiltonian $H^{\prime}$ can be computed by

$$
\begin{aligned}
H^{\prime} & =e^{\chi} H, \\
& =H+\{\chi, H\}+\frac{1}{2}\{\chi\{\chi, H\}\}+\ldots
\end{aligned}
$$

where the symbol $\{$.$\} represents the canonical Poisson brackets. The aim is then to choose carefully \chi$ to eliminate all non-resonant terms in $H^{\prime}$. This can be achieved order by order in $\epsilon$. We expand the function $\chi$ in power of $\epsilon$ as

$$
\chi=\epsilon \chi_{1}+\epsilon^{2} \chi_{2}+\ldots
$$

and we solve order by order in $\epsilon$ the homologic equation for $\chi_{n}$

$$
\left\{\chi_{n}, H_{\text {int }}\right\}+R_{n}=0,
$$

where $H_{\text {int }}$ is given by Eq. (11) and $R_{n}$ gathers all non-resonant terms of order $\epsilon^{n}$ that are created by the Lie transforms up to order $n-1$. The procedure leads to the so-called resonant normal form. The Hamiltonian in resonant normal form only contains terms that can not be integrated out because they are resonant in the accessible domain of phase space. The resonant combination of angles up to third order are displayed in Fig (6).

The computations of the Lie transforms up to order 3 in $\epsilon$ can be done with the special software TRIP. At each order in $\epsilon$ in the Lie transforms, we keep all terms that involve a resonant angle in the accessible domain of phase space. The algorithm gives the Hamiltonian (10) in terms of the new canonical variables

$$
H^{\prime}\left(I^{\prime}, J^{\prime}, \varphi^{\prime}, \psi^{\prime}, t\right)=\widetilde{H}^{\prime}\left(I^{\prime}, J^{\prime}, \varphi^{\prime}, \psi^{\prime}\right)+\epsilon^{3} H_{p e r t}^{\prime}\left(I^{\prime}, J^{\prime}, \varphi^{\prime}, \psi^{\prime}, g t\right)+O\left(\epsilon^{4}\right),
$$


where $\widetilde{H}^{\prime}$ is the autonomous part of the Hamiltonian, and $H_{\text {pert }}^{\prime}$ is the part of the Hamiltonian with all resonant angles of second and third order. The part $H_{\text {pert }}^{\prime}$ has the form

$$
\begin{aligned}
H_{\text {pert }}^{\prime}\left(I^{\prime}, J^{\prime}, \varphi^{\prime}, \psi^{\prime}, g t\right) & =F_{\{2,0,1\}}\left(I^{\prime}, J^{\prime}\right) \cos \left(2 \varphi^{\prime}+g t\right) \\
& +F_{\{2,1,1\}}\left(I^{\prime}, J^{\prime}\right) \cos \left(2 \varphi^{\prime}+\psi^{\prime}+g t\right) \\
& +F_{\{1,2,1\}}\left(I^{\prime}, J^{\prime}\right) \cos \left(\varphi^{\prime}+2 \psi^{\prime}+g t\right) .
\end{aligned}
$$

We have explicitly computed the coefficients $F_{\{2,0,1\}}, F_{\{2,1,1\}}, F_{\{1,2,1\}}$ with TRIP, their explicit expression, together with the expression of $\widetilde{H}^{\prime}$ are available on request to the authors.

\section{B. Explicit expression for $D$}

In the present section, we apply stochastic averaging to the dynamics

$$
\begin{aligned}
\dot{\tilde{H}}^{\prime} & =\left\{H, \widetilde{H}^{\prime}\right\} \\
& =\epsilon^{3}\left\{H_{\text {pert }}^{\prime}, \widetilde{H}^{\prime}\right\} .
\end{aligned}
$$

to find an order of magnitude for the diffusion of $\widetilde{H}^{\prime}$. To simplify the computations and get an explicit expression for the diffusion coefficient, we have chosen reasonable assumptions.

We first notice that the terms of largest amplitude in $\widetilde{H}^{\prime}$ are the terms that depend only on the action variables. To leading order, the expression of $\widetilde{H}^{\prime}$ reduces to

$$
\widetilde{H}^{\prime}\left(I^{\prime}, J^{\prime}, \varphi^{\prime}, \psi^{\prime}\right) \approx H_{i n t}\left(I^{\prime}, J^{\prime}\right),
$$

with the expression of $H_{\text {int }}$ given by Eq. (11).

Using the above approximation in the right-hand side of (16), the dynamics of $\tilde{H}^{\prime}$ reduces to

$$
\dot{\tilde{H}}^{\prime}=-\epsilon^{3} \frac{\partial H_{i n t}}{\partial I^{\prime}} \frac{\partial H_{p e r t}^{\prime}}{\partial \varphi^{\prime}}-\epsilon^{3} \frac{\partial H_{i n t}}{\partial J^{\prime}} \frac{\partial H_{p e r t}^{\prime}}{\partial \psi^{\prime}} .
$$

With the expression (15), Eq. (17) can be rewritten as

$$
\begin{aligned}
\dot{\tilde{H}}^{\prime}\left(I^{\prime}, J^{\prime}, \varphi^{\prime}, \psi^{\prime}, g t\right) & =\bar{F}_{\{2,0,1\}}\left(I^{\prime}, J^{\prime}\right) \sin \left(2 \varphi^{\prime}+g t\right) \\
& +\bar{F}_{\{2,1,1\}}\left(I^{\prime}, J^{\prime}\right) \sin \left(2 \varphi^{\prime}+\psi^{\prime}+g t\right) \\
& +\bar{F}_{\{1,2,1\}}\left(I^{\prime}, J^{\prime}\right) \sin \left(\varphi^{\prime}+2 \psi^{\prime}+g t\right),
\end{aligned}
$$

where $\left\{\bar{F}_{\{2,0,1\}}, \bar{F}_{\{2,1,1\}}, \bar{F}_{\{1,2,1\}}\right\}$ are new coefficients obtained from the expression of $\left\{F_{\{2,0,1\}}, F_{\{2,1,1\}}, F_{\{1,2,1\}}\right\}$. Using stochastic averaging for Eq. (18) (see e.g. [31]), the long-term evolution of $\tilde{H}^{\prime}$ is equivalent in law to a diffusion process

$$
\dot{\tilde{H}}^{\prime}=a\left(\widetilde{H}^{\prime}\right)+\sqrt{D\left(\widetilde{H}^{\prime}\right)} \xi(t) .
$$

The drift term $a\left(\widetilde{H}^{\prime}\right)$ comes from averaging Eq. (18) over fast motion, and from the correlations between fast and slow motion. Numerical simulations done with the dynamics (18) show that the drift is very small compared to the diffusion, and can be neglected, at least in the range of timescale of one billion years we are interested in. In the following, we focus on the diffusion coefficient $D\left(\widetilde{H}^{\prime}\right)$.

The diffusion coefficient can be expressed with a Green-Kubo formula involving the correlation function of the righthand side of Eq. (18). The complete expression is quite long. In this section, in order to get reasonable orders of magnitude, we assume that the cross correlations between different resonant angles give no appreciable contributions. For example, we neglect correlations such as

$$
\left\langle\bar{F}_{\{2,0,1\}}\left(I^{\prime}(t), J^{\prime}(t)\right) \sin \left(2 \varphi^{\prime}(t)+g t\right) \bar{F}_{\{2,1,1\}}\left(I^{\prime}(0), J^{\prime}(0)\right) \sin \left(2 \varphi^{\prime}(0)+\psi^{\prime}(0)\right)\right\rangle .
$$


The functions $\bar{F}\left(I^{\prime}(t), J^{\prime}(t)\right)$ in Eq. (18) can be decomposed between an non-zero averaged part, and a small perturbation with zero average. Clearly, the leading order can be computed retaining only the averaged component of $\bar{F}$. We thus do not longer take into account the dependance on action variables in (18) and we systematically replace the functions $\bar{F}\left(I^{\prime}(t), J^{\prime}(t)\right)$ by a constant corresponding to their order of magnitude. With the approximations discussed above, the order of magnitude for $D\left(\widetilde{H}^{\prime}\right)$ is

$$
\begin{aligned}
D\left(\widetilde{H}^{\prime}\right) & \approx 2\left|\bar{F}_{\{2,0,1\}}\right|^{2} \int_{0}^{+\infty} \mathrm{dt}\langle\sin (2 \varphi(t)+g t) \sin (2 \varphi(0))\rangle_{\widetilde{H}^{\prime}} \\
& +2\left|\bar{F}_{\{2,1,1\}}\right|^{2} \int_{0}^{+\infty} \mathrm{dt}\langle\sin (2 \varphi(t)+\psi(t)+g t) \sin (2 \varphi(0)+\psi(0))\rangle_{\widetilde{H}^{\prime}} \\
& +2\left|\bar{F}_{\{1,2,1\}}\right|^{2} \int_{0}^{+\infty} \mathrm{dt}\langle\sin (\varphi(t)+2 \psi(t)+g t) \sin (\varphi(0)+2 \psi(0))\rangle_{\widetilde{H}^{\prime}}
\end{aligned}
$$

In Eq. (20), the notation $\langle.\rangle_{\widetilde{H}^{\prime}}$ means that the average should be done with a fixed value $\widetilde{H}^{\prime}$.

A last approximation is done to compute the correlation functions of the sinus terms inside the integrals. The two angles $2 \varphi+\psi+g t$ and $\varphi+2 \psi+g t$ correspond to the resonances $2 g_{1}-g_{5}-g_{2}+s_{1}-s_{2}$ and $g_{1}-g_{2}+2\left(s_{1}-s_{2}\right)$ respectively, and are resonant right at the center of the accessible domain as displayed in Fig. (6). Their average frequency is close to zero. On the contrary, the angle $2 \varphi+g t$ is only resonant at the domain boundaries. We choose to keep only the contribution from the last two terms in the right-hand side of Eq. (20). Let $\tau_{L}$ be the correlation time of the angle variables, we choose the approximation

$$
2 \varphi(t)+\psi(t)+g t \approx \varphi(t)+2 \psi(t)+g t \approx \theta+W\left(\frac{t}{\tau_{L}}\right)
$$

where $W(t)$ is the standard Brownian motion and $\theta$ is a random variable with uniform probability distribution over $[0,2 \pi]$. The term $W\left(\frac{t}{\tau_{L}}\right)$ accounts for the fact that a resonant angle crosses the resonant conditions and switches its frequency within a time $\approx \tau_{L}$. We mention that the relation between the Lyapunov exponent of a chaotic Hamiltonian dynamics with one degree of freedom and two resonances has been precisely studied by [33], but the situation with two degrees of freedom is more subtle and the results cannot be directly applied here. The expression (20) for the diffusion coefficient becomes

$$
D \approx 2\left(\left|\bar{F}_{\{2,1,1\}}\right|^{2}+\left|\bar{F}_{\{1,2,1\}}\right|^{2}\right) \int_{0}^{+\infty} \mathbb{E}\left[\sin \left(\theta+W\left(\frac{t}{\tau_{L}}\right)\right) \sin (\theta)\right] \mathrm{d} t .
$$

The computation of the integral in (21) is straightforward. The final result is

$$
D \approx 2\left(\left|\bar{F}_{\{2,1,1\}}\right|^{2}+\left|\bar{F}_{\{1,2,1\}}\right|^{2}\right) \tau_{L}
$$

Finally, we have used the numerical value of the Lyapunov time $\tau_{L} \approx 1.1$ Myr obtained with numerical simulations, and we have evaluated numerically the explicit expressions of $\bar{F}_{\{2,1,1\}}$ and $\bar{F}_{\{1,2,1\}}$. We get the order of magnitude

$$
D \approx 1.15 * 10^{-5} \mathrm{Myr}^{-3}
$$

We further show that the order of magnitude (23) can be obtained in a much more heuristic manner. We have proven that diffusion of the slow variable $\widetilde{H}^{\prime}$ is due to third order secular resonances, that come to order $\epsilon^{3}$ in the Hamiltonian (14). The order of magnitude for $\bar{F}_{\{2,1,1\}}$ and $\bar{F}_{\{1,2,1\}}$ roughly corresponds to $\frac{\left|H_{\text {pert }}\right|^{3}}{|\widetilde{H}|^{3}} \times|\widetilde{H}|^{2}$ and expression $(22)$ can be written

$$
D \approx 2 \frac{\left|H_{p e r t}\right|^{6}}{|\widetilde{H}|^{2}} \tau_{L}
$$

where $|\widetilde{H}|$ is the order of magnitude of the averaged BMH Hamiltonian. Expression (24) corresponds to Eq. (9) of the main text, and direct evaluation with $|\widetilde{H}|=3.10^{-2} \operatorname{arcsec} / \mathrm{yr},\left|H_{\text {pert }}\right| \approx 9 \times 10^{-3} \operatorname{arcsec} / \mathrm{yr}$, and $\tau_{L} \approx 1.1 \mathrm{Myr}$ gives

$$
D \approx 7.2 * 10^{-7} \mathrm{Myr}^{-3}
$$




\section{Destabilization criterion for Mercury's orbit}

We explain in the present section how the stability of Mercury's orbit can be directly related to the value of the slow variable $\widetilde{H}^{\prime}$. $\widetilde{H}^{\prime}$ is obtained by Lie transforms of $\widetilde{H}$ given by (13). The explicit expression of $\widetilde{H}^{\prime}$ is thus composed of a part that depends only on action variables, and a large number of periodic terms that involve the angle variables. The leading terms in the action-dependent part of $\widetilde{H}^{\prime}$ is given by $H_{\text {int }}$. We can thus crudely write the decomposition

$$
\widetilde{H}^{\prime}(I, J, \varphi, \psi, t)=H_{\text {int }}(I, J)+G(I, J, \varphi, \psi, t),
$$

where $G$ is some intricate function. For any fixed value of $\widetilde{H}^{\prime}$, the variations of $H_{\text {int }}$ are bounded between an upper and a lower value

$$
\Gamma_{\text {inf }}\left(\widetilde{H}^{\prime}\right) \leq H_{\text {int }} \leq \Gamma_{\text {sup }}\left(\widetilde{H}^{\prime}\right)
$$

that depend in a non-trivial way of the maximal amplitude of $G(I, J, \varphi, \psi, t)$. The destabilization criterion $H_{i n t}=H_{c r}$ thus translates into the equivalent criterion

$$
\Gamma_{i n f}\left(\widetilde{H}^{\prime}\right)=H_{c r}
$$

Let us call $h_{c r}$ the value such that $\Gamma_{i n f}\left(h_{c r}\right)=H_{c r}$, Mercury's destabilization is directly related to the event $\widetilde{H}^{\prime}=h_{c r}$. This argument shows why destabilization of Mercury is directly related to the event $\widetilde{H}^{\prime}$ hitting the threshold value $h_{c r}$

Given the complexity of the explicit expressions of $\widetilde{H}^{\prime}$ and $\Gamma_{i n f}\left(\widetilde{H}^{\prime}\right)$, the destabilization criterion has to be treated in an empirical manner. The value of $\widetilde{H}^{\prime}$ is better replaced by the local time average $h(t)=\langle\widetilde{H}\rangle_{[t-\theta, t+\theta]}$, where the time frame of length $\theta$ should satisfy $\theta \gg \frac{1}{g_{2}-g_{5}}$. This approximation is described precisely in the main text. In practice, we have chosen $\theta=2 \mathrm{Myr}$. To compute the threshold value $h_{c r}$, we also use a numerical approach: we record the values of $h\left(t_{c r}\right)$ at the destabilization time, for a large number of destabilized trajectories. The distribution of $h\left(t_{c r}\right)$ is peaked at a particular value, thus confirming the existence of the threshold $h_{c r}$. We find $h_{c r} \approx-0.048$ $\operatorname{arcsec} / \mathrm{yr}$.

\section{EXPLICIT EXPRESSION FOR THE DISTRIBUTION OF FIRST EXIT TIMES OF A BROWNIAN MOTION FROM A BOUNDED DOMAIN}

In the present section, we show how to derive the probability distribution function $\rho(\tau)$ of first exit time of a standard Brownian motion from the domain $\left[h_{c r}, h_{\text {sup }}\right]$, starting at $h_{0}$ and with reflective condition at $h=h_{\text {sup }}$.

Let $G(h, t):=\int_{h_{\text {cr }}}^{h_{\text {sup }}} \mathbb{P}\left(h^{\prime}, t \mid h, 0\right) \mathrm{d} h^{\prime}$ be the probability that the Brownian particle starting at $h$ is still in the domain $\left[h_{c r}, h_{\text {sup }}\right]$ at time $t$. It can be shown that the distribution $G(h, t)$ satisfies the same diffusion equation as $\mathbb{P}\left(h^{\prime}, t \mid h, 0\right)$ (see [31])

$$
\frac{\partial G}{\partial t}=D \frac{\partial^{2} G}{\partial h^{2}}
$$

At time $t=0$, the particle is inside the domain, which means that $G(h, 0)=1$ for all $h \in\left[h_{c r}, h_{s u p}\right]$. The absorbing boundary condition at $h=h_{c r}$ and the reflecting boundary condition at $h=h_{\text {sup }}$ can be equivalently expressed with the distribution $G$ as

$$
\text { for all } t>0, \begin{cases}G\left(h_{c r}, t\right) & =0, \\ \frac{\partial G}{\partial h}\left(h_{\text {sup }}, t\right) & =0 .\end{cases}
$$

We solve the problem (25-26) by decomposing the solution into proper modes. Let us introduce the standard scalar product

$$
\langle f, g\rangle=\frac{2}{h_{\text {sup }}-h_{c r}} \int_{h_{c r}}^{h_{\text {sup }}} f(h) g(h) \mathrm{d} x .
$$


It can be checked that the family of functions

$$
e_{n}(h)=\cos \left(\pi\left(n+\frac{1}{2}\right) \frac{h-h_{\text {sup }}}{h_{c r}-h_{\text {sup }}}\right) \text { with } n \in \mathbb{N}
$$

form an orthonormal basis of all functions $G(x, t)$ satisfying the boundary conditions (26). The solution of (25-26) can thus be expressed as the Fourier series

$$
G(h, t)=\stackrel{[}{n}=0]+\infty \sum g_{n}(t) e_{n}(h),
$$

where the coefficients $g_{n}(t)$ are defined as the projection of $G$ on the orthonormal basis, that is $g_{n}(t):=\left\langle G(h, t) e_{n}(h)\right\rangle$. Using the Fourier decomposition (27), we find that $G$ is solution of (25) if and only if

$$
g_{n}(t)=g_{n}(0) e^{-\pi^{2}\left(n+\frac{1}{2}\right)^{2} \frac{D}{\left(h_{\text {sup }}-h_{c r}\right)^{2}} t} .
$$

The value $g_{n}(0)$ can be found with the initial condition $G(h, 0)=1$. We get

$$
g_{n}(0)=\left\langle G(h, 0) e_{n}(h)\right\rangle=\frac{2}{\pi} \frac{(-1)^{n}}{n+\frac{1}{2}} .
$$

Finally, the solution $G(h, t)$ can be expressed explicitly as

$$
\left.G(h, t)=\frac{2}{\pi} \stackrel{[}{n}=0\right]+\infty \sum \frac{(-1)^{n}}{n+\frac{1}{2}} \cos \left(\pi\left(n+\frac{1}{2}\right) \frac{h-h_{\text {sup }}}{h_{c r}-h_{\text {sup }}}\right) e^{-\pi^{2}\left(n+\frac{1}{2}\right)^{2} \frac{D}{\left(h_{\text {sup }}-h_{c r}\right)^{2}} t} .
$$

As $G(h, t)$ is the probability to be still in the domain $\left[h_{c r}, h_{s u p}\right]$ at time $t$, it is related to $\rho(\tau)$ by

$$
G(h, t)=\int_{t}^{+\infty} \rho(\tau) \mathrm{d} \tau .
$$

Therefore, the time derivative of Eq. (28) gives the explicit expression of $\rho(\tau)$

$\left.\rho(\tau)=\frac{2 \pi D}{\left(h_{\text {sup }}-h_{c r}\right)^{2}} \stackrel{[}{n}=0\right]+\infty \sum(-1)^{n}\left(n+\frac{1}{2}\right) \cos \left(\pi\left(n+\frac{1}{2}\right) \frac{h_{0}-h_{c r}}{h_{\text {sup }}-h_{c r}}\right) \exp \left(-\pi^{2}\left(n+\frac{1}{2}\right)^{2} \frac{D \tau}{\left(h_{\text {sup }}-h_{\text {cr }}\right)^{2}}\right)^{2}$

This expression is used for the fit in Fig. (4) of the main text.

\section{AVERAGE AND VARIANCE OF A BROWNIAN BRIDGE}

In the present section, we show how to obtain explicitly the red curves in Fig. (5) of the main text.

The aim is to compute the probability

$$
\rho^{\tau_{e x}}(h, t):=\mathbb{P}\left(h, t \mid\left\{h_{0}, 0\right\} \cap\left\{\tau=\tau_{e x}\right\}\right)
$$

to have a trajectory at location $h$ at time $t$ with the constrains that the trajectory starts at $h_{0}$ and exits the domain at time $\tau=\tau_{e x}$, for a standard Brownian motion of diffusion coefficient $D$. The inequality $0<t<\tau_{e x}$ should be satisfied. Using Bayes theorem and Markov property, the probability distribution (30) can be written as

$$
\rho^{\tau_{e x}}(h, t)=\frac{\mathbb{P}\left(\tau=\tau_{e x} \mid h, t\right) \mathbb{P}\left(h, t \mid h_{0}, 0\right)}{\mathbb{P}\left(\tau=\tau_{e x} \mid h_{0}, 0\right)} .
$$

All probability distributions in the right-hand side of (31) have explicit expressions. The probability $\mathbb{P}\left(\tau=\tau_{\text {ex }} \mid h, t\right)$ to exit the domain starting at a given position can be obtained from equation (29) in the limit $h_{\text {sup }} \rightarrow+\infty$. We have thus

$$
\begin{aligned}
\mathbb{P}\left(\tau=\tau_{e x} \mid h, t\right) & =\frac{1}{\tau_{e x}-t} \frac{h-h_{c r}}{\sqrt{4 \pi D\left(\tau_{e x}-t\right)}} e^{-\frac{\left(h-h_{c r}\right)^{2}}{4 D\left(\tau_{e x}-t\right)}}, \\
\mathbb{P}\left(\tau=\tau_{e x} \mid h_{0}, 0\right) & =\frac{1}{\tau_{e x}} \frac{h_{0}-h_{c r}}{\sqrt{4 \pi D \tau_{e x}}} e^{-\frac{\left(h_{0}-h_{c r}\right)^{2}}{4 D \tau_{e x}}} .
\end{aligned}
$$


The last term $\mathbb{P}\left(h, t \mid h_{0}, 0\right)$ is simply the solution of the free diffusion equation in an infinite domain, which is the classical result

$$
\mathbb{P}\left(h, t \mid h_{0}, 0\right)=\frac{1}{\sqrt{4 \pi D t}} e^{-\frac{\left(h-h_{0}\right)^{2}}{4 D t}} .
$$

After some algebra, we obtain the following explicit expression for $\rho^{\tau_{e x}}(h, t)$ (valid for $h>h_{c r}$ and $0<t<\tau_{e x}$ )

$$
\begin{aligned}
\rho^{\tau_{e x}}(h, t) & =\frac{h-h_{c r}}{h_{0}-h_{c r}}\left(\frac{\tau_{e x}}{\tau_{e x}-t}\right)^{3 / 2} \frac{1}{\sqrt{4 \pi D t}} \\
& \times\left\{\exp \left(-\frac{\left(h-h_{c r}-(1-s)\left(h_{0}-h_{c r}\right)\right)^{2}}{4 D \tau_{e x} s(1-s)}\right)-\exp \left(-\frac{\left(h-h_{c r}+(1-s)\left(h_{0}-h_{c r}\right)\right)^{2}}{4 D \tau_{e x} s(1-s)}\right)\right\}
\end{aligned}
$$

where we have introduced the ratio $s=\frac{t}{\tau_{e x}}$. It can be quite easily checked that $\int_{h_{c r}}^{+\infty} \rho^{\tau_{e x}}(h, t) \mathrm{d} h=1$, because $\rho^{\tau_{e x}}(h, t)$ is a probability density.

The instanton trajectory, and the variance of the distribution around the instanton can be obtained with the first and the second moments of the distribution (32). We define the average trajectory $\{\bar{h}(t)\}_{0<t<\tau_{e x}}$ as

$$
\bar{h}(t)=\int_{h_{c r}}^{+\infty} h \rho^{\tau_{e x}}(h, t) \mathrm{d} h .
$$

There is a small difference between the average trajectory defined by (33) and the instanton trajectory $\widetilde{h}(t)$ which is the trajectory of highest probability. The trajectory of highest probability is the straight trajectory of equation

$$
\widetilde{h}(t)=\frac{t}{\tau_{e x}} h_{c r}+\left(\frac{\tau_{e x}-t}{\tau_{e x}}\right) h_{0} .
$$

The distribution of trajectories that exit the domain for short times is more and more concentrated around the trajectory of highest probability when $\tau_{e x}$ goes to zero. To first approximation, $\widetilde{h} \approx \bar{h}$ when $\tau_{e x}$ is small compared to $\tau^{*}$. However, the average trajectory is a bit curved when $t$ gets closer to $\tau_{e x}$ because of the influence of the absorbing boundary condition. We represent in Fig. (5) of the main text the averaged trajectory instead of the instanton trajectory because it can more easily be compared to numerical results. To study the trajectories dispersion around the instanton, we can also compute the standard deviation

$$
\delta \bar{h}(t)=\left[\int_{h_{c r}}^{+\infty}(h-\bar{h}(t))^{2} \rho^{\tau_{e x}}(h, t) \mathrm{d} h\right]^{1 / 2} .
$$

Expressions (33) and (34) can be evaluated numerically. The three red curves in Fig. (5) of the main text are those of equations (from highest to lowest) $h(t)=\bar{h}(t)+\delta \bar{h}(t), h(t)=\bar{h}(t)$ and $h(t)=\bar{h}(t)-\delta \bar{h}(t)$ respectively, for $\tau_{e x}=445$ million years.

\section{INFORMATION ABOUT THE NUMERICAL SIMULATIONS}

\section{A. Figure 4}

The probability distribution of Mercury's first destabilization time represented by the blue curve in Fig. 4 of the main text has been obtained from a direct numerical simulation of Hamilton's equations

$$
\left\{\begin{array}{l}
\dot{\varphi}(t)=\frac{\partial H}{\partial I} \\
\dot{\psi}(t)=\frac{\partial H}{\partial J}
\end{array}\right.
$$

where $H$ is given by Eq. (10). We used a Runge-Kutta scheme of order 4 . We integrated $N=126518$ trajectories with initial conditions uniformly chosen in the range $\left[\varphi_{0}-10^{-2}, \varphi_{0}+10^{-2}\right]$. The simulation is stopped either when the trajectory reaches $I=0.06$ or when the time of integration becomes larger than $T=3.1$ billion years. We recorded $n=57330$ trajectories that have reached $I=0.06$ before the maximal integration time. We recall that $I=0.06$ 
means that the trajectory has entered the unbounded part of phase space and the orbit can therefore be considered as destabilized.

Then, the blue curve of Fig. (4) of the main text is obtained by fitting the distribution of the $n$ recorded times with the function "kernel" of matlab, with the normalization set to one. To compare the result with the diffusion model, we plotted in red in Fig (4) of the main text the expression $\rho(\tau) * N / n$ with $\rho(\tau)$ given by Eq. $(29)$ and $D=9.6 * 10^{-7}$ $M y r^{-3}$.

\section{B. Estimation of the drift coefficient}

In this section, we give an estimation of the drift $a\left(\widetilde{H}^{\prime}\right)$ in Eq. (19). The aim is to show that $a\left(\widetilde{H}^{\prime}\right)$ can be neglected to compute Mercury's first destabilization time with the diffusion model. For this purpose, we do the following numerical simulation: we integrate Hamilton's equations for the dynamics defined by the Hamiltonian of Eq. (4) of the main text

$$
\widetilde{H}=H_{i n t}+E_{2} \sqrt{I} \cos (\varphi)+S_{2} \sqrt{J} \cos (\psi) .
$$

We record $N=1000$ trajectories starting from initial conditions chosen uniformly in the range $\left[\varphi_{0}-10^{-2}, \varphi_{0}+10^{-2}\right]$, and for a time $T=206 \mathrm{Myr}$. Note that those trajectories are necessarily bounded because the value of $\widetilde{H}$ is conserved. Then we compute the explicit expression of $\dot{\widetilde{H}}(I, J, \varphi, \psi, t)=\left\{\widetilde{H}, H_{\text {pert }}\right\}$, and we use it to integrate the equation

$$
\dot{F}=\dot{\widetilde{H}}(I(t), J(t), \varphi(t), \psi(t), t)
$$

where $(I(t), J(t), \varphi(t), \psi(t))$ is a trajectory computed previously. $F(t)$ can be seen as a good approximation for $\widetilde{H}^{\prime}(t)-\widetilde{H}^{\prime}(0)$ at short times. We obtain this way a set of $N=1000$ trajectories $\left\{F_{i}(t)\right\}$.

Finally, we plot on Fig. (7) the histogram of the $\left\{F_{i}(t)\right\}$ for $t=41 / 82 / 123 / 165 / 206$ Myr, and we fit the different histograms with the Gaussian distribution

$$
\rho(F)=\frac{1}{\sqrt{2 \pi \sigma^{2}(t)}} e^{-\frac{(F-m(t))^{2}}{2 \sigma^{2}(t)}} .
$$

We observe that the quantity $\sigma^{2}(t)$ indeed scales linearly with $t$, as expected for a diffusion process. The value of $m(t)$ is non vanishing because of the short-term oscillations of $\widetilde{H}$ on the Myr timescale. To obtain a relevant order of magnitude for the long-term drift, we have to subtract the shift due to the short-term oscillations. The quantity

$$
A=\max \left\{m(t)-m\left(t^{\prime}\right) ; t, t^{\prime} \in[0, T]\right\} / T \approx 1.1 * 10^{-6} M y r^{-2}
$$

gives us an order of magnitude for the drift $a\left(\widetilde{H}^{\prime}\right)$ in Eq. (19). Correspondingly, we find that the error due to the drift, for $t=3000$ Myr should not exceed $A t \approx 3.3 * 10^{-3} M_{y r^{-1}}$. With $D \approx 10^{-6} M_{y r}{ }^{-3}$, we find that the variation of $\widetilde{H}^{\prime}(t)$ due to the diffusion coefficient over the same timescale is of the order of $\sqrt{D t} \approx 5 * 10^{-2} M_{y r}^{-1}$. We conclude that there is one order of magnitude between the respective effects of the drift term and the diffusion term in Eq. (19), and that the former can be neglected on the billion years timescale. 


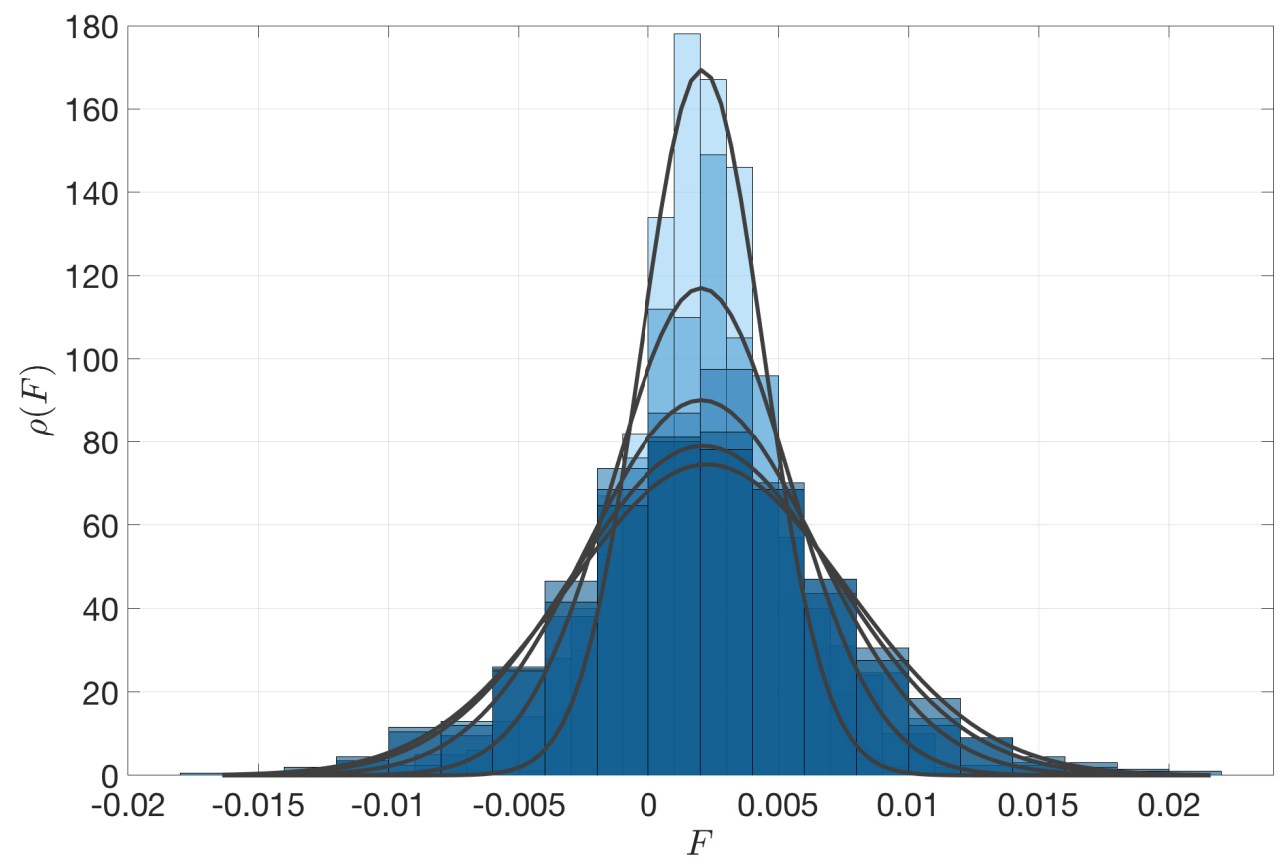

FIG. 7: The distribution of $F(t)$ for different times $t=41 / 82 / 123 / 165 / 206$ Myr. The blue histograms represent the distributions of the $N=1000$ trajectories generated by Eq. (V B). The darkness of the histogram increases with time. The grey curves represent the Gaussian fits of the histograms. The average and variance of those distributions give us order of magnitudes for the drift and the diffusion coefficient of the diffusion model for $\widetilde{H}^{\prime}$. To leading order, the drift can be neglected. 